\title{
GALAXY FORMATION AND CLUSTER FORMATION
}

\author{
Richard B. Larson \\ Yale Astronomy Department
}

\section{INTRODUCTION}

A primary motivation for studying globular clusters is that, as the oldest known galactic fossils, they trace the earliest stages of galactic evolution; indeed, they may hold the key to understanding galaxy formation. Thus it is clearly of great importance to learn how to read the fossil record. To do this, we need to understand something about how the globular clusters themselves formed. Were they the first bound objects to form, or did they form in larger pre-existing systems of which they are just small surviving fragments? If the latter, what were the prehistoric cluster-forming systems like? And how did they manage to produce objects like globular clusters?

\section{CLUES TO CLUSTER FORMATION}

Several pieces of evidence indicate that most globular clusters formed very early. The ages of the well-studied galactic globular clusters (VandenBerg, this symposium; Demarque, this symposium) imply that these clusters formed within at most the first few Gyr of the history of the universe. In addition, as has been noted by Harris (1986; this symposium), the globular clusters in elliptical galaxies generally have a more extended spatial distribution and a flatter radial density profile than the background stars; in the brightest ellipticals the density distribution of the globular clusters follows approximately $\mathrm{r}^{-2 \cdot 5}$, compared with $\mathrm{r}^{-3 \cdot 0}$ for the stars. The globular clusters are also generally bluer and thus presumably more metal-poor than the field stars at the same distance from the center in elliptical galaxies (Harris 1983). These properties suggest that the globular clusters formed earlier than the field stars in these galaxies, since in any picture of galaxy formation involving progressive condensation and enrichment of the gas, a population that forms earlier has both a lower metallicity and a flatter density profile (Larson 1969). 
However, there is also evidence that globular clusters are not primordial objects but formed only after considerable star formation activity had already taken place. The radial distribution of the globular clusters in elliptical galaxies, while flatter than that of the stars, is steeper than the $\mathrm{r}^{-2}$ profile usually assumed for the dark matter in galactic halos, suggesting that the dark halos formed first; if the dark matter is in faint stars or stellar remnants that originated in an early Population III, the globular clusters formed after the Population III objects. More compelling evidence that globular clusters are not primordial is provided by the fact that, with very few exceptions, they are internally very homogeneous in metallicity: the dispersion in measured stellar $[\mathrm{Fe} / \mathrm{H}]$ values in a cluster is less than 0.15 (Norris, this symposium), and the narrowness of the giant branch in M3 implies a dispersion in [ $\mathrm{Fe} / \mathrm{H}]$ less than 0.04 (Buonanno et al., this symposium). This is a much smaller spread in metallicity than has been found in any system of galactic size, including the dwarf spheroidal galaxies (Da Costa, this symposium). The gas from which a cluster formed must therefore have been thoroughly mixed, presumably by turbulence generated by energy input from massive stars, subsequent to its chemical enrichment but before the formation of the observed stars. This implies that the pre-cluster gas was retained in a bound star-forming system long enough for turbulence to homogenize it before the cluster formed. In order to retain the gas, such a system must almost certainly have been much more massive than the cluster itself, implying that globular clusters formed only as small parts of much larger star-forming systems (e.g. Searle 1977, Searle and Zinn 1978).

If the formation of globular clusters is at all similar to the formation of open clusters in our Galaxy, observations of nearby regions of star formation also suggest that bound clusters form only as small parts of much larger complexes of gas and young stars. For example, in Orion there is a small and very dense grouping of young stars immediately surrounding the Trapezium that will probably survive as a bound open cluster (Herbig and Terndrup 1986), but it contains only a few hundred solar masses, compared with a total of several thousand solar masses of young stars and several times $10^{5}$ solar masses of gas in the whole Orion region of star formation. Thus only about $10^{-3}$ of the mass in this region seems likely to end up in a bound star cluster. If a similarly low efficiency applies to the formation of globular clusters, this suggests that they originated in star-forming systems or complexes with masses exceeding $10^{8}$ solar masses. It may be relevant that the smallest galaxy known to contain globular clusters, the Fornax dwarf spheroidal galaxy, has a present mass of about $2 \times 10^{7}$ solar masses, and it may well have had a much larger initial mass.

\section{SITES OF CLUSTER FORMATION}

What did the primitive cluster-forming systems look like? The 
above considerations suggest that they typically had the masses of dwarf galaxies, and some of them may have been much larger. Indeed, since there is a population of globular clusters associated with the old disk of our Galaxy (Zinn 1985; this symposium), globular clusters can evidently form in the disks of large spiral galaxies, at least early in their evolution. Thus globular clusters may have formed in systems with masses anywhere between perhaps $10^{8}$ and $10^{11}$ solar masses. Most presently observed gas-rich, actively star-forming galaxies in this mass range are rotationally flattened disks, so many globular clusters may actually have formed in disks. Most of these early disk systems must since have been destroyed by collisions or mass loss that left them unbound and dispersed their stellar contents.

Since the field stars in elliptical galaxies may well also have formed in disk systems that later merged (Toomre 1977), and since most of the stars in elliptical galaxies are probably quite old, the early disk systems must have formed their stars quite rapidly, probably mostly within the first few Gyr. They must therefore have been more compact than typical present-day irregular or spiral galaxies, since a higher star formation rate requires a higher mean density (Larson 1977). Typical irregular or spiral galaxies also do not make clusters as massive as typical globular clusters, so they cannot serve as suitable prototypes for the birthplaces of globular clusters. The original star-forming systems must have been both more gas-rich and more compact than present-day galaxies, and they must have formed stars more vigorously and in larger complexes. If observed today, such a system would probably be called a blue compact or starburst galaxy.

A similar picture is suggested by the available evidence on the circumstances in which very massive star clusters form. The disk of our Galaxy evidently stopped forming globular clusters early in its history, and now forms only much less massive open clusters. The Large Magellanic Cloud is currently forming more massive clusters, as is M33 (Christian, this symposium), but these clusters are still perhaps an order of magnitude less massive than typical galactic globular clusters. As in our Galaxy, the young clusters in these galaxies are less massive than the old ones, and only the oldest LMC clusters approach typical galactic globular clusters in size. The most luminous young cluster in the Local Group, located in the bright LMC HII region 30 Doradus, has a total luminosity of about $4 \times 10^{7}$ suns (Jones et al. 1986) and contains about three hundred 0 stars (Moffat et al. 1985); with a conventional IMF, this implies a total stellar mass of about $10^{5}$ solar masses. However, nothing is known directly about low-mass stars in 30 Dor, so it cannot be predicted how much mass will remain in a bound cluster; probably it will not exceed a few times $10^{4}$ solar masses. The resulting cluster may then have up to a hundred times the mass of the Trapezium cluster, but it will still fall at least an order of magnitude short of being a good candidate for a young globular cluster, which should have at least a thousand times the mass of the Trapezium cluster. The total mass of 
gas in the 30 Dor region is about $5 \times 10^{7}$ solar masses (Rohlfs et al. 1984), also roughly a hundred times the total mass in the Orion region (Maddalena et a1. 1986) rather than the three orders of magnitude more that may be required to form a globular cluster.

Starburst galaxies may be more promising sites for the formation of globular clusters. The nearby prototype M82, which is currently experiencing a vigorous burst of star formation in a compact nuclear gas disk, contains a number of bright stellar knots with luminosities exceeding $10^{8}$ suns (van den Bergh 1971) that could represent, or contain, young globular clusters. Again, however, nothing is known about the low-nass stellar content or the spatial structure of these knots. Another system of interest is $I \mathrm{Zw} \mathrm{18}$, which contains a very bright HII region that has a luminosity of order $10^{8}$ suns, an estimated stellar mass of $10^{6}$ solar masses, and a metal abundance $[\mathrm{Fe} / \mathrm{H}]$ of -1.5 (Lequeux and Viallefond 1980). The most massive young cluster known may be the "super star cluster" in NGC 1705 (Melnick et a1. 1986), which has a luminosity of $2 \times 10^{8}$ suns but contains no 0 stars, implying an age of at least $10^{7}$ years. Its inferred mass is then about $5 \times 10^{6}$ solar masses, assuming a conventional IMF, although this assumption may not be valid in starburst galaxies (Larson 1987). In any case it would appear that the conditions required for globular cluster formation are at least approached, if not met, in some starburst galaxies.

Similar conditions may exist in some of the giant HII regions in the outer parts of the giant spiral galaxy M101, which typically contain several million solar masses of young stars (Viallefond et al. 1981). If sufficiently massive bound clusters exist in these luminous HII regions, the conditions required for globular cluster formation may be met or approached even in some late-type giant spirals. If so, it may be that the essential requirement for forming globular clusters is simply a very gas-rich system with active star formation occurring in very massive complexes.

\section{THE MECHANISM OF CLUSTER FORMATION}

How, then, do globular clusters form in the larger, flattened star-forming systems that have been discussed? And can one account for the fact that very massive clusters seem to form only in youthful, gas-rich, vigorously star-forming systems, while the typical mass of the clusters that form in a galaxy decreases with time as the galaxy ages?

The massive gas complexes in which star clusters are born must be self-gravitating, and their formation almost certainly involves gravity acting on large scales. A mechanism that is probably of very general importance for star formation is the gravitational instability of a galactic gas layer or disk; this can play a role not only in generating spiral structure in galaxies (Goldreich and Lynden-Be11 
1965, Toomre 1981) but also in initiating or organizing star formation in them (Larson 1977, 1983). In a typical spiral galaxy like our own, gravitational instability of the gas layer produces structures with typical sizes of a few $\mathrm{kpc}$ and masses of several times $10^{7}$ solar masses, comparable to a major spiral arm segment or to the 30 Dor complex in the LMC. Thus gravitational instability can account for at least the large-scale structure of major star-forming regions in our Galaxy and others (Elmegreen and Elmegreen 1983). Further

gravitational condensation processes on smaller scales, which are not yet well understood, are evidently required to form the small dense cloud cores in which star clusters and ultimately individual stars are born. The development and fragmentation of flattened or filamentary structures may play an important role also in these later stages of cloud condensation (Larson 1985).

The stability of a rotating gas disk is determined by the parameter $Q=c k / \pi G \mu$, where $c$ is the velocity dispersion of the gas, $\mu$ is its surface density, and $K$ is the epicyclic frequency. In a thin disk, violent instability occurs when $Q<1$; limited growth of spiral density perturbations occurs for $1<Q<\sim 2$ (Larson 1984); and stability occurs for $Q>\sim 2$. Numerical simulations of disks containing gas (e.g. Sellwood and Carlberg 1984) show that, while dissipation tends to reduce $Q$ and make the disk more unstable, the ensuing instabilities tend to raise the velocity dispersion again and maintain $Q$ at a nearly constant value of about 2 .

If the gas layer in a disk galaxy is thus maintained in a state of marginal stability throughout its evolution, the typical size and mass of any structures formed by gravitational instability will decrease with time as the gas is depleted by star formation. This is because, if the epicyclic frequency $\kappa$ does not change much, a constant value of $Q$ implies that the velocity dispersion $c$ of the gas decreases as the gas surface density $\mu$ decreases, and this results in smaller length scales for instability (e.g. Carlberg and Freedman 1985). In the solar neighborhood, for example, the gas surface density may have decreased by as much as an order of magnitude from the time of disk formation to the present, implying a similar order-or-magnitude decrease in the velocity dispersion of the gas (if the contribution of stars to the instability of the gas layer is neglected.) The typical mass of structures formed by gravitational instability varies as $c^{4} / \mu$ (Larson 1985), so if both $\mu$ and $c$ decrease by an order of magnitude, the predicted mass scale decreases by three orders of magnitude. This is just the factor by which the Trapezium cluster (which is relatively sma11) must be scaled up in mass to make a typical globular cluster. Thus it may be possible to understand in this way why globular clusters formed only during the early evolution of the Milky Way disk, and why the presently forming clusters are much less massive.

This conclusion probably does not depend crucially on the assumption that the gas layer remains marginally stable at all times. Even if the velocity dispersion of the gas is much too small to 
maintain stability, there is still a maximum length scale on which instability can operate that is proportional to $\mu / \mathrm{k}^{2}$. If this maximum length scale is identified with the size of the largest star-forming complexes that develop, which are probably the birthplaces of the most massive clusters, then the mass of these largest complexes is predicted to vary as $\mu^{3}$, just as is predicted by the above argument for "typical" structures.

Although not intended to represent the formation of star clusters, several simulations of the fragmentation of gas disks (Larson 1978, Wood 1982, Miyama et al. 1984) show results that are at least qualitatively consistent with the predictions of gravitational instability theory as outlined above. In particular, they show that when the velocity dispersion (temperature) of the gas is decreased, the disk becomes thinner and the typical size and mass of the fragments formed is reduced. The simulations of Larson (1978) also show that very dense cores can form and will eventually accrete most of the mass if no disruptive effects operate. Thus, in addition to gravitational instability, accretional growth of condensations in a galactic disk may play a role in the development of massive clouds and of dense cluster-forming cores within them (see also Larson 1983). Effects such as ionization by massive stars may then limit how massive these cluster-forming cores can become before they self-destruct.

Some evidence that settling of the galactic gas layer occurred early in the evolution of the Milky Way disk and was accompanied by a decrease in the size of the clusters formed is provided by the fact that the luminosities of the disk globular clusters in our Galaxy appear to increase with distance from the galactic plane (Zinn 1985); for example, the disk clusters more than $500 \mathrm{pc}$ from the plane are on the average about $1.3 \mathrm{mag}$ more luminous than those closer to the plane.

\section{STAR FORMATION IN GLOBULAR CLUSTERS}

In order for a bound globular cluster to be produced, a cluster-forming cloud must form low-mass stars with high efficiency. Also, if the protocluster gas is not to be ionized and dispersed by massive stars before most of its has condensed into low-mass stars, it is probably necessary for star formation to be completed in a time shorter than stellar evolution timescales, i.e. shorter than $10^{6}$ years. Both of these requirements may be satisfied if cluster-forming cloud cores are dense enough. Very high densities must indeed have existed, since the observed densities of globular clusters, allowing for some expansion due to stellar mass loss, imply that their birth clouds must have had remarkably high average densities of at least $10^{5}$ molecules per $\mathrm{cm}^{3}$.

It has often been suggested that the earliest star formation in galaxies produced mostly or exclusively massive stars: for example, 
the initial enrichment of halo stars and their high oxygen-to-iron ratio have been attributed to an early generation of massive stars, and it has been suggested that the early formation of mostly massive stars is to be expected because of the relatively high gas temperature (Larson 1986). Also, a variety of cosmological phenomena may be explainable by postulating large numbers of pregalactic very massive stars (Carr et al. 1984). If early star formation favored massive stars, then globular clusters must have been exceptional objects, since star formation in them evidently produced mostly low-mass stars.

The fragmentation process and the resulting stellar IMF in a star-forming cloud depend critically on the temperature-density relation: fragmentation can continue as long as the gas temperature decreases with increasing density, but it becomes less likely and may stop altogether when the temperature stops decreasing and begins to increase with increasing density (Larson 1985). In a region of very active star formation, the dust is heated by the strong infrared radiation field present (Falgarone and Puget 1985), and this causes the gas temperature to turn upward with increasing density when the critical mass for fragmentation is approximately 5 solar masses (Larson 1987). This suggests that in starburst regions the formation of stars less massive than several solar masses is inhibited, as has also been suggested on observational grounds. However, the formation of low-mass stars can still occur in exceptionally dense cloud cores where the density exceeds $10^{6}$ molecules per $\mathrm{cm}^{3}$; in such regions the critical mass becomes less than one solar mass despite the elevated temperature (Larson 1987). Thus a globular cluster can form in a massive cloud core in which most of the gas has a density of $10^{6}$ molecules per $\mathrm{cm}^{3}$ or more. In nearby molecular clouds, only very small regions with masses of several tens or hundreds of solar masses have such high densities, and this suggests again that the formation of globular clusters requires much more massive clouds.

If a protocluster cloud core has an average density of $10^{6}$ molecules per $\mathrm{cm}^{3}$, its free-fall time is only $3 \times 10^{4}$ years, so it is possible that star formation is largely completed within $10^{5}$ years, i.e. well before any of the stars begin to evolve and before ionization has eroded away much of the gas. However, most of the gas will probably have been ionized and dispersed by the time supernovae begin to explode in the cluster after about $10^{7}$ years (Tenorio-Tagle et al. 1986). For this reason, chemical self-enrichment of globular clusters seems unlikely to occur.

Another implication of the required very high initial density of globular clusters is that at this density, the dissolution time for a globular cluster of typical mass is of the same order as the Hubble time. For a fixed density, the dissolution time of a cluster is proportional to its mass; thus globular clusters with much smaller masses should not have survived to the present time, and this may explain why such clusters are not now observed. 
Clearly many properties of globular clusters remain to be explained, including most of those discussed at this symposium. Not only cluster formation processes but also destruction mechanisms will clearly be important in accounting for the properties of globular clusters and for their correlations with the environment. Given the current rudimentary state of theorizing on the formation of globular clusters, it will be difficult to make any very quantitative predictions about how they began; indeed, it seems enough of a challenge to understand the mere existence of these remarkable objects.

\section{REFERENCES}

Carlberg, R. G. and Freedman, W. L. 1986 Astrophys. J. 298, 486. Carr, B. J., Bond, J. R. and Arnett, W. D. 1984 Astrophys. J. 277,445 .

Elmegreen, B. G. and Elmegreen, d. M. 1983 Monthly Notices Roy. Astron. Soc. 203, 31 .

Falgarone, E. and Puget, J. L. 1985 Astron. Astrophys. 142, 157.

Goldreich, P. and Lynden-Be11, D. 1965 Monthly Notices Roy. Astron. Soc. $130,125$.

Harris, W. E. 1983 Publ. Astron. Soc. Pacific 95, 21.

Harris, W. E. 1986 Astron. J. 91, 822.

Herbig, G. H. and Terndrup, D. M. 1986 Astrophys. J. 307, 609.

Jones, T. J., Hyland, A. R., Straw, S., Harvey, P. M., Wilking, B. A., Joy, M., Gatley, I. and Thomas, J. A. 1986 Monthly Notices Roy. Astron. Soc. 219, 603 .

Larson, R. B. 1969 Monthly Notices Roy. Astron. Soc. 145, 405.

Larson, R. B. 1977 in The Evolution of Galaxies and Stellar

Populations B. M. Tinsley and R. B. Larson, eds., Yale University Observatory, New Haven, p. 97.

Larson, R. B. 1978 Monthly Notices Roy. Astron. Soc. 184, 69.

Larson, R. B. 1983 Highlights of Astronomy 6, 191.

Larson, R. B. 1984 Monthly Notices Roy. Astron. Soc. 206, 197.

Larson, R. B. 1985 Monthly Notices Roy. Astron. Soc. 214, 379.

Larson, R. B. 1986 Monthly Notices Roy. Astron. Soc. 218, 409.

Larson, R. B. 1987 in Stellar Populations C. A. Norman, A. Renzini, and M. Tosi, eds., Cambridge University Press, in press.

Lequeux, J. and Viallefond, F. 1980 Astron. Astrophys. 91, 269.

Maddalena, R. J., Morris, M., Moscowitz, J. and Thaddeus, P. 1986 Astrophys. J. 303, 375.

Melnick, J., Moles, M. and Terlevich, R. 1985 Astron. Astrophys. $149, \mathrm{~L} 24$.

Moffat, A. F. J., Seggewiss, W. and Shara, M. M. 1985 Astrophys. J. $295,109$.

Miyama, S. M., Hayashi, C. and Narita, S. 1984 Astrophys. J. 279, 621.

Rohlfs, K., Kreitschmann, J., Siegman, B. C. and Feitzinger, J. V. 1984 Astron. Astrophys. 137, 343. 
Searle, L. 1977 in The Evolution of Galaxies and Stellar Populations

B. M. Tinsley and R. B. Larson, eds., Yale University Observatory, New Haven, p. 219.

Searle, L. and Zinn, R. 1978 Astrophys. J. 225, 357.

Sellwood, J. A. and Carlberg, R. G. 1984 Astrophys. J. 282, 61.

Tenorio-Tagle, G., Bodenheimer, P., Lin, D. N. C. and Noriega-Crespo,

A. 1986 Monthly Notices Roy. Astron. Soc. 221, 635.

Toomre, A. 1977 in The Evolution of Galaxies and Stellar Populations

B. M. Tinsley and R. B. Larson, eds., Yale University Observatory, New Haven, p. 401.

Toomre, A. 1981 in The Structure and Evolution of Normal Galaxies

S. M. Fall and D. Lynden-Bell, eds, Cambridge University Press,

p. 111 .

van den Bergh, S. 1971 Astron. Astrophys. 12, 474.

Viallefond, F., Allen, R. J. and Goss, W. M. 1981 Astron. Astrophys. $104,127$.

Wood, D. 1982 Monthly Notices Roy. Astron. Soc. 199, 331.

Zinn, R. J. 1985 Astrophys. J. 293, 424. 


\section{DISCUSSION}

GRINDLAY: If globular clusters are indeed formed in gaseous disks by instabilities, how do you account for the clusters systems in giant ellipticals such as M 87? What are the implications for formation of the ellipticals themselves?

LARSON: I would suggest that giant ellipticals form by mergers of disk systems of various sizes. There is now good enough observational evidence to make a convincing case that at least some elliptical galaxies form or are built up by mergers of disk galaxies. The globular clusters in ellipticals probably form as a result of starburst activity during the early evolution of such disks, while the field stars mostly form somewhat later in these disks when the metallicity has increased and globular clusters have stopped forming.

OSTRIKER: If globular clusters are all made via gravitational instabilities in disks, why is the system so spherical now? If they were made in preexisting disk systems that merged, then how did the clusters know how to arrange themselves so that the more metal-rich clusters became more centrally concentrated in our Galaxy?

LARSON: I imagine that spherical globular cluster systems form from subsystems that merge with little total angular momentum, although the subsystems may individually be flattened and rotating. The entire proctology probably had some overall organization, perhaps provided by the potential well of a dark halo, and leftover gas continued to dissipate and settle toward the center as it became chemically enriched. I am suggesting that, in order to form globular clusters, the protogalaxy contained internal structure consisting of flattened star-forming subsystems.

MATHIEU: I have two questions regarding star formation in globular clusters. First, do you think that the apparent paucity of binaries in globular clusters indicates differences in the star formation processes relative to those of Pop I stars and Pop II field stars and do you have any conjecture as to why these differences might arise? And second, how many supernovae would be required to remove the parent gas of a globular cluster? Does the presence of hundreds of 0 stars suggest greater coevality $\left(<10^{6} \mathrm{yr}\right)$ than that seen in newly star forming regions $\left(-10^{7} \mathrm{yr}\right)$ ?

LARSON: I know of no reason why globular clusters should have formed relatively few binaries; maybe most of the original binaries in globular clusters were destroyed. The chances for destruction might be increased if the clusters were initially much denser. Supernovae are probably not a very important mechanism of cloud destruction because ionization by 0 stars is likely to have done even greater damage before supernovae began to explode, after $-10^{7}$ years. Of course one wants to avoid destruction by ionization before most of the low-mass stars have formed, but at the densities of $>10^{6} \mathrm{~cm}^{-3}$ that I suggest for 
proto-globular clusters the free-fall time is only a few times $10^{4}$ years. So star formation may indeed occur within only a very short time interval.

VAN DEN BERGH: It seems to me that the correlations of metallicity, cluster radius and the second parameter of globular clusters with galactocentric distance suggest that the proto-galaxy must already have been quite highly organized at the time clusters were formed. There is also a qualitative difference between the formation of clusters in the Magellanic Clouds and in the protogalaxy: (1) clusters in the clouds have larger $\xi$ than their galactic counterparts and (2) the mass spectrum and globular clusters differs from that with which clusters are formed in the clouds.

LARSON: I agree that there must have been some overall organization, as I indicated in my answer to Ostriker. I would also reiterate that the star-forming, subsystems that made the globular clusters could not have been just like typical present irregular or spiral galaxies, which are too loose in structure and form stars at too leisurely a pace. Thus the Magellanic Clouds are not a good prototype. More compact, gas-rich, and actively star forming systems containing exceedingly massive gas complexes are required, and the closest present analogies are more likely to be starburst galaxies.

BHATIA: How do you explain the formation of sparse clusters like Pal 14 which exist in the halo of the Galaxy?

LARSON: I don't pretend to be able to predict the structure of globular clusters when they form. Maybe a great many sparse clusters were formed initially, and only those at large galactocentric distances have survived. A possible way of making sparse clusters from initially dense ones would be as a result of stellar mass loss in a system whose initial mass function contained a large proportion of massive stars. Maybe the dwarf spheroidal galaxies are very loose because they lost a lot of mass in this way.

RICHER: Can you form Jupiter's (few thousandths of a $\mathrm{M}_{V}$ ) in your star formation picture in globular clusters?

LARSON: My feeling is that it would be difficult to make many Jupiters in globular clusters. To make Jupiters you need very low temperatures and/or exceedingly high densities. I think that fragmentation to smaller masses continues effectively as long as the temperature continues to decrease with increasing density, but becomes much less likely when the temperature stops decreasing and begins to rise again. In nearby cold dark clouds, this allows one to understand the formation of objects as small as a few tenths of a solar mass, but suggests that Jupiters are much harder to form. Maybe they only form in dense pre-planetary disks around stars like the Sun. 